including heading and non-heading exercises; they also completed two regular football sessions. For each accelerative event recorded, PLA, PRA and PRV outputs were compared to video recordings. Receiver operating characteristic curves were used to determine the sensor's discriminatory capacity in both on-field settings, determining cut-off values for predicting outcomes.

Results For the laboratory tests, the random error was $11 \%$ for PLA, 20\% for PRA and 5\% for PRV, respectively; the systematic error was $11 \%, 19 \%$ and $5 \%$. For the structured training protocol, heading events yielded higher absolute values $(\mathrm{PLA}=15.6 \pm 11.8 \mathrm{~g}$ ) than non-heading events (PLA=4.6 $\pm 1.2 \mathrm{~g}$ ); the area under the curve (AUC) was 0.98 for PLA. In regular training sessions, AUC was $>0.99$ for PLA. A $9 \mathrm{~g}$ cut-off value yielded a positive predictive value of $100 \%$ in the structured training protocol, compared to only $65 \%$ in regular football sessions.

Conclusion The sensor displayed systematic overestimation with considerable random error. Despite excellent on-field accuracy for discriminating head-impacts from other accelerative events, secondary means of verifying events are still necessary.

\section{EVALUATION OF IN-EAR SENSOR SYSTEMS FOR QUANTIFYING HEAD IMPACT EXPOSURE IN YOUTH FOOTBALL}

${ }^{1}$ Stian Bahr Sandmo*, 2,3,4Andrew S Mclntosh, 'Thor Einar Andersen, 5,6Inga K Koerte, ${ }^{1}$ Roald Bahr. 'Department of Sports Medicine, Oslo Sports Trauma Research Center, Norwegian School of Sport Sciences, Norway; ${ }^{2}$ Federation University Australia, Australia; ${ }^{3}$ Monash University Accident Research Centre, Monash University, Australia; ${ }^{4}$ McIntosh Consultancy and Research, Australia; ${ }^{5}$ Department of Child and Adolescent Psychiatry, Psychosomatic and Psychotherapy, Ludwig-Maximilian University, Germany; ${ }^{6}$ Department of Psychiatry, Pscyhiatry Neuroimaging Laboratory, Brigham and Women's Hospital, Harvard Medical School, USA

\subsection{6/bjsports-2019-scandinavianabs.24}

Introduction Wearable sensor systems may be useful for measuring head-impact exposure. Here, we tested the validity of in-ear sensors developed to improve head coupling.

Methods First, the sensor was mounted to a Hybrid III headform (HIII) and impacted with a linear impactor or football. Peak linear acceleration (PLA), peak rotational acceleration (PRA) and peak rotational velocity (PRV) were obtained from both systems; random and systematic error were calculated using HIII as reference. Then, six youth football players wore sensors and performed a structured training protocol including heading and non-heading exercises; they also completed two regular football sessions. For each accelerative event recorded, PLA, PRA and PRV outputs were compared to video recordings. Receiver operating characteristic curves were used to determine the sensor's discriminatory capacity in both on-field settings, determining cut-off values for predicting outcomes.

Results For the laboratory tests, the random error was $11 \%$ for PLA, 20\% for PRA and 5\% for PRV, respectively; the systematic error was 11\%, 19\% and 5\%. For the structured training protocol, heading events yielded higher absolute values $(\mathrm{PLA}=15.6 \pm 11.8 \mathrm{~g}$ ) than non-heading events (PLA=4.6 $\pm 1.2 \mathrm{~g}$ ); the area under the curve (AUC) was 0.98 for PLA. In regular training sessions, AUC was $>0.99$ for PLA. A $9 \mathrm{~g}$ cut-off value yielded a positive predictive value of $100 \%$ in the structured training protocol, compared to only $65 \%$ in regular football sessions.
Conclusion The sensor displayed systematic overestimation with considerable random error. Despite excellent on-field accuracy for discriminating head-impacts from other accelerative events, secondary means of verifying events are still necessary.

\section{INCREASED TTTG MEASURED ON AXIAL MRI. IS IT DUE TO LATERALIZATION OF THE TIBIAL-TUBERCLE OR MEDIALIZATION OF THE TROCHLEAR-GROOVE?}

${ }^{1}$ Mathias Paiva, ${ }^{2}$ Lars Blønd, ${ }^{1}$ Per Hölmich, ${ }^{1}$ Kristoffer Barfod*. ${ }^{1}$ Sports Orthopedic Research Center Copenhagen (SORC-C), Department of Orthopedic, Danmark; 'Zealand University Hospital, Koge, Danmark

\subsection{6/bjsports-2019-scandinavianabs.25}

Introduction To investigate if increased Tibial tubercle - Trochlear Groove distance (TT-TG) measured on axial MRI is due to lateralization of the tibial tubercle or medialization of the trochlear groove.

Methods 65 knees (28 normal (NK), 25 with trochlear dysplasia (TD) and 12 with patellar dislocation without TD (PD)) were examined. The medial border of the posterior cruciate ligament (PCL) was chosen as the central anatomical landmark. The distance from the tibial tubercle (TT) to PCL (TTPCL) was measured to examine the lateralization of the tibial tubercle. The distance from the trochlear groove to PCL (TGPCL) was measured to examine the medialization of the trochlear groove. Between group differences was investigated by use of one-way ANOVA.

Results The mean (SD) values for TT-TG were $8.5 \mathrm{~mm}$ (3.6) for NK, $11.4 \mathrm{~mm} \mathrm{(6.2)} \mathrm{for} \mathrm{PD} \mathrm{and} 17.1 \mathrm{~mm} \mathrm{(4.8)}$ in the TD group $(\mathrm{p}<0.01)$. The mean $(\mathrm{SD})$ values for TT-PCL were $19.5 \mathrm{~mm}$ (4.2) for NK, $17.0 \mathrm{~mm} \mathrm{(5.0)} \mathrm{for} \mathrm{PD} \mathrm{and} 20.2 \mathrm{~mm}$ (5.0) in the TD group $(p=0.10)$. The mean (SD) values for TG-PCL were $10.5 \mathrm{~mm}$ (3.7) for NK, $5.8 \mathrm{~mm} \mathrm{(4.9)} \mathrm{for} \mathrm{PD}$ and $3.9 \mathrm{~mm}(3.9)$ in the dysplastic group $(\mathrm{p}<0.01)$.

Conclusion TD knees had increased TT-TG compared to NK and PD. The TT-PCL distance did not differ significantly between groups, whereas the TG-PCL distance declined with increased TT-TG. The present results indicate that increased TT-TG is due to medialization of the trochlear groove and not lateralization of the tibial tubercle.

\section{AFTER SIX-WEEKS OF INTENSIVE BALLET REHEARSALS CHANGES IN THE ACHILLES TENDONS STRUCTURE APPEAR WHEREAS SELF-REPORTED SEVERITY SCORES REMAIN THE SAME}

\begin{abstract}
${ }^{1,2}$ Charlotte Anker-petersen*, ${ }^{2}$ Kristian Thorborg, ${ }^{3}$ Jarrod Antflick, ${ }^{4}$ Henrik Aagaard, ${ }^{3}$ Chris Myers, ${ }^{2}$ Anders Ploug Boesen, ${ }^{2}$ Per Hølmich, ${ }^{1}$ Birgit Juul-Kristensen. ${ }^{1}$ Department of Sports Science and Clinical Biomechanics, University of Southern Denmark, Denmark; ${ }^{2}$ Sports Orthopedic Research Center - Copenhagen; Department of Orthopedic Surgery, Amager-Hvidovre Hospital, Denmark; ${ }^{3}$ Tendon Performance, UK; ${ }^{4}$ Department of Orthopedic Surgery, Sjaelland University Hospital, Denmark
\end{abstract}

\subsection{6/bjsports-2019-scandinavianabs.26}

Introduction The aim was to study whether six weeks of intensive dance exposure (Swan Lake rehearsals) is a significant contributor to structural changes, symptoms, clinical signs and pain in the Achilles tendon (AT).

Materials and methods Ballet dancers from The Royal Danish Ballet Company (aged 18-41) were invited $(n=79)$ of which 
sixty-three $(80 \%)$ dancers participated. Baseline scans were collected with follow-up scans at six weeks. The primary outcome was quantification of AT structure with Ultrasound Tissue Characteristics (UTC) (echo-type I-IV). Secondary outcomes of interest were clinical signs and symptoms gathered from a clinical examination, self-reported symptoms, VISA-A questionnaire and pain during single-legged heel raise. UTC has previously shown to have satisfactory reproducibility and validity. Separate multilevel linear and logistic regression models were performed including time and demographic variables as covariates.

Result From baseline to follow-up there was significant decrease in distribution of UTC echo-type I $(\beta=-3.6$, $\mathrm{p}=0.001 ; 95 \% \mathrm{CI}:-5.8 ;-1.5)$ with significant increase in echotype II $(\beta=3.2, p<0.0001 ; 95 \% \mathrm{CI}: 1.6 ; 4.8)$. Significant effects were also seen, of limb (type I+III) and gender (type I+II). No significant changes were found in clinical outcomes/clinical signs and symptoms.

Conclusion The cohort of ballet dancers showed significant UTC changes, mainly a reduction of echo-type I distribution after six-weeks pre-season period rehearsing Swan Lake ballet. No changes were found in clinical outcomes/clinical signs and symptoms. However, early structural changes seem important to follow longitudinally for potential planning of secondary prevention strategies.

\section{INCIDENCE OF SPORTS-RELATED INJURIES AND ILLNESSES IN PARALYMPIC ATHLETES - A PROSPECTIVE STUDY DURING 52 WEEKS}

${ }^{1}$ Kristina Fagher* ${ }^{*}$ 2,3 Örjan Dahlström, ${ }^{2}$ Jenny Jacobsson, ${ }^{2}$ Toomas Timpka, ${ }^{1,4}$ Jan Lexell. ${ }^{1}$ Department of Health Sciences, Rehabilitation Medicine Research Group, Lund University, Sweden; ${ }^{2}$ Department of Medical and Health Sciences, Athletics Research Center, Linköping University, Sweden; ${ }^{3}$ Department of Behavioural Sciences and Learning, Linköping University, Sweden; ${ }^{4}$ Department of Neuroscience, Rehabilitation Medicine, Uppsala University, Sweden

\subsection{6/bjsports-2019-scandinavianabs.27}

Introduction Sports-related injuries and illnesses in Paralympic sport (SRIIPS) is a growing concern, but knowledge about the etiology of SRIIPS is limited. The aim of this study was to prospectively assess the incidence and risk factors of SRIIPS among Swedish Paralympic athletes during 52 weeks.

Materials and methods 107 Paralympic athletes weekly reported SRIIPS in an eHealth application adapted to Paralympic athletes. Descriptive statistics and the Kaplan Meier method with corresponding Cox proportional hazards regression analysis $(p<0.05)$ was used to assess incidence, time to SRIIPS, survival probability and hazard ratio (HR).

Results The incidence of injuries and illnesses was 7.0/1000 and 9.5/1000 hours of sport exposure, respectively. For injury the survival probability was $31.8 \%$ and median time to injury was 19 weeks (95\% CI: 10.6-27.4). A higher risk to sustain an injury was noted among athletes; $\geq 30$ years (HR 1.6; 95\% CI: 1.01-2.53), with previous severe injury (HR 2.4; 95\% CI: 1.47-3.83), in team sports (HR 1.8; 95\% CI: 1.10-2.80) and males (HR 1.8; 95\% CI: 1.062.93). For illness, the survival probability was $23.4 \%$. Median time to illness was 9 weeks (95\% CI: 1.4-16.6). Athletes reporting an injury (HR 1.8; 95\% CI: 1.08-2.98) and participants in team sports (HR 1.6; 95\% CI: $1.05-$ 2.54) had a higher risk for illness.
Conclusion This is the first longitudinal long-term prospective study of SRIIPS. Males, older athletes, athletes in team sports and with previous incidents are particularly at risk for injuries and illnesses and should therefore be targets for preventive measures and future research.

\section{INCREASED HIP ADDUCTION DURING RUNNING IS ASSOCIATED WITH PATELLOFEMORAL PAIN AND DIFFERS BETWEEN MALES AND FEMALES: A CASE- CONTROL STUDY}

\begin{abstract}
${ }^{1,2}$ Bradley Neal ${ }^{*}{ }^{3,4}$ Christian Barton, ${ }^{1,5}$ Aleksandra Birn-Jeffrey, ${ }^{1,6}$ Dylan Morrissey. ${ }^{1}$ Sports and Exercise Medicine, Queen Mary University of Londoon, UK; ${ }^{2}$ Pure Sports Medicine, UK; ${ }^{3}$ La Trobe Sport and Exercise Medicine Research Centre, Australia; ${ }^{4}$ School of Allied Health, La Trobe University, Australia; ${ }^{5}$ School of Engineering and Materials Science, Queen Mary University of Londoon, UK; ${ }^{6}$ Physiotherapy Department, Barts Health NHS Trust, UK
\end{abstract}

\subsection{6/bjsports-2019-scandinavianabs.28}

Introduction Patellofemoral pain (PFP) is common amongst recreational runners and associated with altered running kinematics. However, it is currently unclear how sex may influence kinematic differences previously reported in runners with patellofemoral pain. This case-control study aimed to evaluate lower limb kinematics in males and females with and without patellofemoral pain during prolonged running.

Materials and methods Lower limb 3D kinematics were sampled in 20 runners with PFP (11 females, 9 males) and 20 asymptomatic runners (11 females, 9 males) during a $3 \mathrm{~km}$ treadmill run. Data were analysed when mean-pooled as mixed sex groups (PFP versus control) and as individual sex sub-groups.

Results Mixed-sex runners with PFP were found to have significantly greater peak hip adduction (mean difference $=4.9^{\circ}$, $\mathrm{d}=0.91, \quad 95 \%$ CI 1.4-8.2, $\mathrm{p}=0.01$ ) when compared to matched controls. Analyses for all other kinematic variables were non-significant. Females with PFP ran with greater peak hip adduction compared to female controls (mean difference $=6.6^{\circ}, \mathrm{p}=0.02, \mathrm{~F}=3.41,95 \%$ CI $0.4-12.8$ ), but not males with or without PFP. Analyses of sub-group comparisons for all other kinematic variables were non-significant.

Conclusion Differences in peak hip adduction between those with and without PFP during running appear to be driven by female participants, highlighting potentially different kinematic treatment targets for the individual sexes. Future research is encouraged to report lower limb kinematic variables in runners with PFP separately for males and females.

\section{IS TWO-DIMENSIONAL VIDEO A VALID AND RELIABLE MEASURE OF THREE-DIMENSIONAL KINEMATICS IN RUNNERS WITH PATELLOFEMORAL PAIN?}

\footnotetext{
1,2 Bradley Neal* ${ }^{1,2}$ Simon Lack, ${ }^{3,4}$ Christian Barton, ${ }^{1,5}$ Aleksandra Birn-Jeffrey ${ }^{1}$ Stuart Miller, ${ }^{1,6}$ Dylan Morrissey. ${ }^{1}$ Sports and Exercise Medicine, Queen Mary University of London, UK; ${ }^{2}$ Pure Sports Medicine, UK; ${ }^{3}$ La Trobe Sport and Exercise Medicine Research Centre, La Trobe University, Australia; ${ }^{4}$ School of Allied Health, La Trobe University, Australia; ${ }^{5}$ School of Engineering and Materials Science, Queen Mary University of London, UK; ${ }^{6}$ Physiotherapy Department, Queen Mary University of London, UK
}

\subsection{6/bjsports-2019-scandinavianabs.29}

Introduction Peak hip adduction (HADD) and knee flexion (KFLEX) during running are associated with patellofemoral pain (PFP) persistence, representing treatment targets. Clinical 